\title{
A FOCAL BOUNDARY VALUE PROBLEM FOR DIFFERENCE EQUATIONS
}

\author{
CATHRYN DENNY and DARREL HANKERSON \\ Department of Algebra, Combinatorics, and Analysis \\ Auburn University \\ Auburn, Alabama 36849-5307
}

(Received August 27, 1991 and in revised form April 11, 1992)

\begin{abstract}
The eigenvalue problem in difference equations, $(-1)^{n-k} \Delta^{n} y(t)=\lambda \sum_{i=0}^{k-1} p_{i}(t) \Delta^{i} y(t)$, with $\Delta^{i} y(0)=0,0 \leq i<k, \Delta^{k+i} y(T+1)=0,0 \leq i<n-k$, is examined. Under suitable conditions on the coefficients $p_{i}$, it is shown that the smallest positive eigenvalue is a decreasing function of $T$. As a consequence, results concerning the first focal point for the boundary value problem with $\lambda=1$ are obtained.
\end{abstract}

KEY WORDS AND PHRASES. Difference equation, eigenvalue, boundary value problem, focal point, Green's function.

1991 MATHEMATICS SUBJECT CLASSIFICATION CODES. 39A10.

\section{INTRODUCTION}

Let $k$ and $n$ be integers with $1 \leq k<n$. For functions $y$ defined on an interval of integers, define the difference operator $\Delta$ by $\Delta^{0} y=y, \Delta y(t)=y(t+1)-y(t)$, and $\Delta^{i} y=\Delta\left(\Delta^{i-1} y\right)$ for $i \geq 1$. We shall be concerned first with the eigenvalue problem for difference equations

$$
\begin{aligned}
& (-1)^{n-k} \Delta^{n} y(t)=\lambda \sum_{i=0}^{k-1} p_{i}(t) \Delta^{i} y(t), \quad 0 \leq t \leq T \\
& \Delta^{i} y(0)=0, \quad 0 \leq i \leq k-1, \\
& \Delta^{k+i} y(T+1)=0, \quad 0 \leq i \leq n-k-1
\end{aligned}
$$

where $T$ is a nonnegative integer. Throughout this paper, the interval notation in expressions such as (1.1) denote intervals of integers; for example, $[0, T]=\{0,1, \ldots, T\}$. Under suitable conditions on the coefficients $p_{i}$, we show that the smallest positive eigenvalue is a decreasing function of $T$.

Next, we will consider the boundary value problem (1.2),

$$
(-1)^{n-k} \Delta^{n} y(t)=\sum_{i=0}^{k-1} p_{i}(t) \Delta^{i} y(t), \quad 0 \leq t \leq T
$$

If there is a nontrivial solution of (1.3), (1.2), then $T$ is said to be a $(k, n-k)$-focal point of (1.3). The smallest such $T$ is called the first $(k, n-k)$-focal point, or, more briefly, the first focal point. The results concerning the monotonicity of the eigenvalue will be used to investigate relationships between the existence of first $(k, n-k)$-focal points of (1.3) and the existence of solutions of (1.3), (1.2) that are positive with respect to a cone in a suitable Banach space. 
It can be shown that the Green's function $G_{T}(t, s)$ for the focal boundary value problem

$$
\begin{aligned}
& (-1)^{n-k} \Delta^{n} y(t)=\delta_{t s} \\
& \Delta^{2} y(0)=0, \quad 0 \leq i \leq k-1, \\
& \Delta^{k+z} y(T+1)=0, \quad 0 \leq i \leq n-k-1,
\end{aligned}
$$

exists. Extensive discussions concerning Green's functions for difference equations can be found in Hartman [1] and Kelley and Peterson [2]; see also [3]. In particular, if $y(t)$ is a solution of (1.3), (1.2) on $[0, T+n]$, then $y(t)$ solves the equation

$$
y(t)=\sum_{s=0}^{T} G_{T}(t, s) \sum_{i=0}^{k-1} p_{i}(s) \Delta^{i} y(s), \quad t \in[0, T+n] .
$$

As a consequence, if sign conditions on $G_{T}(t, s)$ are known and certain positivity conditions are placed on the $p_{i}$ 's, then questions concerning the eigenvalue of $(1.1),(1.2)$ and the existence of focal points for (1.3), (1.2) can be examined in terms of a family of linear operators that depend on $T$.

Many authors have applied the theory of cones in a Banach space and positive operators either to demonstrate the existence of smallest positive eigenvalues, to compare these eigenvalues, or to establish the existence of first conjugate points or first focal points of boundary value problems for linear equations; see, for example, Eloe and Henderson [4, 5], Gentry and Travis [6], Hankerson and Henderson [7], Hankerson and Peterson [8, 9], Keener and Travis [10], Tomastik [11, 12], and Travis [13]. We also mention papers by Eloe [14] and Eloe and Henderson [15] which examine criteria for disfocality of difference equations, two papers by Henderson $[16,17]$ on focal boundary value problems for nonlinear difference equations, and a paper by Henderson and Lee [18] on continuous dependence and differentiation of solutions of difference equations. Much of our motivation for this study are the works of Keener and Travis [19], Schmitt and Smith [20], and Tomastik [21].

In section 2, we include preliminary notation, and fundamental results from the theory of cones in a Banach space. In section 3, we show, under suitable assumptions on the coefficients $p_{i}$, that the smallest positive eigenvalue of (1.1), (1.2) decreases with $T$. This will lead to results concerning the first focal point of (1.3), (1.2).

\section{PRELIMINARIES}

In this section, we give definitions and auxiliary results from cone theory. Much of the discussion in this section involving the theory of cones in a Banach space arises from results in Krasnosel'skii's book [22]. Other good references include Kreĭn and Rutman [23], and Deimling [24].

Let $B$ be a Banach space. A closed subset $K$ of $B$ is said to be a cone provided: (i) if $u, v \in K$ then $\alpha u+\beta v \in K$ for all $\alpha, \beta \geq 0$, and (ii) if $u,-u \in K$ then $u=0$. A cone $K$ is said to be reproducing provided every $x \in B$ can be written as $x=u-v$ for some $u, v \in K$. If $K$ is a cone and $u, v \in B$, then we write $u \leq v$ (with respect to $K$ ) provided $v-u \in K$. If $L$ and $M$ are linear operators on $B$, then $L \leq M$ (with respect to $K$ ) provided $L y \leq M y$ for all $y \in K$. Finally, given a bounded linear operator $L$ on $B$, we say that $L$ is positive if $L(K) \subseteq K$, and we say that $L$ is $u_{0}$-positive if given any nonzero $u \in K$, there exist $k_{1}, k_{2}>0$, such that $k_{1} u_{0} \leq L u \leq k_{2} u_{0}$. If $L: B \rightarrow B$ is a bounded linear operator, we shall use $r(L)$ to denote the spectral radius of $L$.

THEOREM 2.1 Let $L$ be a positive compact linear operator with respect to a reproducing cone $K$ and let $r(L)>0$. Then $r(L)$ is an eigenvalue of $L$ with corresponding eigenvector in $K$. 
THEOREM 2.2 If $L$ and $M$ are compact linear positive operators such that $L \leq M$, then $r(L) \leq$ $r(M)$.

THEOREM 2.3 Let $L$ be a compact linear positive operator, and suppose $L x \geq \mu x$ for some $\mu>0$ and $x \in B$ with $-x \notin K$ and $x=u-v$ for some $u, v \in K$. Then $L$ has an eigenvector $x_{0} \in K$ corresponding to an eigenvalue $\lambda_{0} \geq \mu$.

Theorems 2.5, 2.10, 2.11, and 2.13 of Krasnosel'skin give the following theorem.

THEOREM 2.4 Let $K$ be a reproducing cone. If $L$ is a compact $u_{0}$-positive linear operator then $L$ has an essentially unique eigenvector in $K$ and the corresponding eigenvalue is simple, positive, and larger than the modulus of any other eigenvalue.

The following theorem appears in Keener and Travis [19, Theorem 2.3] and is a generalization of Travis [13, Theorem 2.3].

THEOREM 2.5 Let $L$ and $M$ be bounded linear operators and assume that at least one of the operators is $u_{0}$-positive. Assume $L \leq M$ and there exist nonzero vectors $u_{1}, u_{2}, \in K$ and scalars $\lambda_{1}, \lambda_{2}>0$ such that $L u_{1} \geq \lambda_{1} u_{1}$ and $M u_{2} \leq \lambda_{2} u_{2}$. Then $\lambda_{1} \leq \lambda_{2}$. If $\lambda_{1}=\lambda_{2}$ then $u_{1}$ is a scalar multiple of $u_{2}$.

\section{EIGENVALUES AND FOCAL POINTS}

Our main objective in this section is to describe how the smallest positive eigenvalue of (1.1), (1.2) changes with $T$. We will transform these questions about the eigenvalue into questions about the spectral radius of certain operators on a Banach space, and then apply the cone theory.

First, let $(c)^{(i)}$ denote the factorial polynomial defined by $(c)^{(i)}=c(c-1) \cdots(c-i+1)$. If $G_{T}(t, s)$ is the Green's function for (1.4), then

$$
\Delta^{j} G_{T}(t, s)=\sum_{\tau=0}^{\min \{t-1, s\}} \frac{(t-\tau-1)^{(k-j-1)}}{(k-j-1) !} \frac{(-\tau+s+n-k-1)^{(n-k-1)}}{(n-k-1) !}
$$

for $j<k$, and

$$
\Delta^{k+j} G_{T}(t, s)= \begin{cases}\frac{(-1)\}(s+n-k-1-(t+j))(n-k-1-\jmath)}{(n-k-1-j) !}, & t \leq s \\ 0, \quad t>s & \end{cases}
$$

for $0 \leq j \leq n-k-1$. The Green's function can be found with the aid of [25, Lemma 1].

Next, let $B=\left\{y:[0, \infty) \rightarrow \mathbf{R} \mid y\right.$ is bounded and $\left.\Delta^{i} y(0)=0,0 \leq i<k\right\}$. Then $B$ is a Banach space under the sup norm. Define $L_{T}: B \rightarrow B$ by

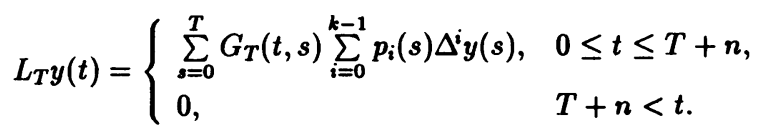


We assume that $p_{2}(t) \geq 0$ for $t \geq 0,0 \leq i \leq k-1$.

Remark. Note that $L_{T} y$ depends only on the values of $y$ on $[0, T+k-1]$. Hence, if $T_{1} \geq T$ and $B_{T_{1}}=\left\{y:\left[0, T_{1}+k-1\right] \rightarrow \mathbb{R} \mid \Delta^{z} y(0)=0,0 \leq i \leq k-1\right\}$, then we can regard $L_{T}$ as a map $B_{T_{1}} \rightarrow B$. We can then define the map $M_{T}: B_{T_{1}} \rightarrow B_{T_{1}}$ by $M_{T} z=\left.L_{T} z\right|_{\left[0, T_{1}+k-1\right]}$.

Now, if $(\lambda, z)$ is an eigenpair for $M_{T}$, then we can extend $z$ to a function in $B$ by setting $y(t)=z(t)$ for $0 \leq t \leq T_{1}+k-1$ and $\lambda y(t)=L_{T} z(t)$ for $t \geq T_{1}+k$; then $(\lambda, y)$ is an eigenpair for $L_{T}$. Conversely, if $(\lambda, y)$ is an eigenpair for $L_{T}$ with $\lambda \neq 0$, then $z=\left.y\right|_{\left[0, T_{1}+k-1\right]} \neq 0$ (otherwise $L_{T} y=0$ and $\left.\lambda=0\right)$ and $(\lambda, z)$ is an eigenpair for $M_{T}$.

In addition, for any function $x(t)$ defined on $[0, T+k-1]$, the expression $L_{T} x$ makes sense and we will allow this slight abuse of notation. Then if $y(t)=L_{T} x(t)$, it follows that $y(t)$ is a solution of the boundary value problem (1.2),

$$
(-1)^{n-k} \Delta^{n} y(t)=\sum_{i=0}^{k-1} p_{i}(t) \Delta^{i} x(t)
$$

If $(\lambda, x)$ is an eigenpair for $L_{T}$ with $\lambda \neq 0$, then $(1 / \lambda, x)$ is an eigenpair for (1.1), (1.2), and conversely. Note also that $\lambda=0$ is not an eigenvalue of (1.1), (1.2).

To begin with, we wish to examine, under suitable conditions, what happens to the spectral radius of $L_{T}$ as $T$ increases. Our first result will play a key role in subsequent work.

THEOREM 3.1 The spectral radius $r\left(L_{T}\right)$ is a nondecreasing function of T. Morever, if $p_{i_{0}}(T)>$ 0 for some $T \geq k-i_{0}$, then $r\left(L_{T-1}\right)<r\left(L_{T}\right)$.

PROOF. Define the cone $K_{T}$ in $B_{T}$ by

$$
K_{T}=\left\{y \in B_{T} \mid \Delta^{i} y(t) \geq 0, t \in[0, T+k-1-i], 0 \leq i \leq k-1\right\}
$$

Then $K_{T}^{\circ}=\left\{y \in B_{T} \mid \Delta^{i} y(t)>0, t \in[k-i, T+k-1-i], 0 \leq i \leq k-1\right\}$, and $K_{T}$ is reproducing.

We will begin by showing $r\left(L_{T}\right)$ is nondecreasing; that is, we will show $r\left(L_{T-1}\right) \leq r\left(L_{T}\right)$. Let $x \in K_{T}$ and regard $L_{T-1}$ as an operator on $B_{T}$. Then

$$
\begin{aligned}
\Delta^{k} L_{T-1} x(t) & =\sum_{s=t}^{T-1} \frac{(s+n-k-1-t)^{(n-k-1)}}{(n-k-1) !} \sum_{i=0}^{k-1} p_{i}(s) \Delta^{i} x(s) \\
& \leq \sum_{s=t}^{T} \frac{(s+n-k-1-t)^{(n-k-1)}}{(n-k-1) !} \sum_{i=0}^{k-1} p_{i}(s) \Delta^{i} x(s) \\
& =\Delta^{k} L_{T} x(t), \quad 0 \leq t \leq T-1 .
\end{aligned}
$$

We can repeatedly sum both sides of $\Delta^{k} L_{T-1} x(t) \leq \Delta^{k} L_{T} x(t)$ and use the boundary conditions to show that $\Delta^{i} L_{T-1} x(t) \leq \Delta^{i} L_{T} x(t)$ for $0 \leq t \leq T+k-1-i, 0 \leq i \leq k-1$. Then $L_{T-1} \leq L_{T}$ with respect to $K_{T}$ and by Theorem $2.2, r\left(L_{T-1}\right) \leq r\left(L_{T}\right)$.

Now suppose that $p_{i_{0}}(T)>0$ for some $T \geq k-i_{0}$. We first show that $r\left(L_{T}\right)>0$. Let $u(t)=t^{(k)} \in K_{T}^{\circ}$. Note that

$$
\Delta^{\prime} L_{T} u(t)=\sum_{s=0}^{T} \Delta^{\prime} G_{T}(t, s) \sum_{i=0}^{k-1} p_{i}(s) \Delta^{i} u(s) \geq \Delta^{j} G_{T}(t, T) p_{i_{0}}(T) \Delta^{i_{0}} u(T)>0
$$


for $k-j \leq t \leq T+k-j-1$ and $0 \leq j \leq k-1$. Thus, $L_{T} u \in K_{T}^{\circ}$. It follows that there exists an $\epsilon>0$ such that $L_{T} u \geq \epsilon u$, and by Theorem 2.3 we have $r\left(L_{T}\right) \geq \epsilon>0$.

Finally, we will show $r\left(L_{T-1}\right)<r\left(L_{T}\right)$. Assume $r\left(L_{T-1}\right)>0$. By Theorem 2.1, $r\left(L_{T-1}\right)$ is an eigenvalue of $L_{T-1}$ with corresponding eigenvector $y \in K_{T-1}$. We can use $r\left(L_{T-1}\right) y=L_{T-1} y$ to extend $y$ to a function in $B_{T}$. We claim that $y \in K_{T}^{\circ}$. To see this, since $y$ is an eigenvector corresponding to $r\left(L_{T-1}\right)$, there exists $s_{0} \in[0, T-1]$ such that $\sum_{i=0}^{k-1} p_{i}\left(s_{0}\right) \Delta^{i} y\left(s_{0}\right)>0$. Then

$$
\begin{aligned}
r\left(L_{T-1}\right) \Delta y(t) & =\sum_{s=0}^{T-1} \Delta^{\prime} G_{T-1}(t, s) \sum_{i=0}^{k-1} p_{i}(s) \Delta^{i} y(s) \\
& \geq \Delta G_{T-1}\left(t, s_{0}\right) \sum_{i=0}^{k-1} p_{i}\left(s_{0}\right) \Delta^{i} y\left(s_{0}\right)>0
\end{aligned}
$$

for $t \in[k-j, T+k-1-j], 0 \leq j \leq k-1$. It follows that $y \in K_{T}^{\circ}$. Hence $p_{t_{0}}(T) \Delta^{i_{0}} y(T)>0$ and

$$
\begin{aligned}
\Delta^{k} L_{T-1} y(t) & =\sum_{s=0}^{T-1} \Delta^{k} G_{T-1}(t, s) \sum_{i=0}^{k-1} p_{i}(s) \Delta^{i} y(s) \\
& <\sum_{s=0}^{T} \Delta^{k} G_{T}(t, s) \sum_{i=0}^{k-1} p_{i}(s) \Delta^{i} y(s) \\
& =\Delta^{k} L_{T} y(t), \quad 0 \leq t \leq T-1
\end{aligned}
$$

Using the boundary conditions at 0 , we obtain $L_{T} y-L_{T-1} y \in K_{T}^{\circ}$. Hence, there exists an $\epsilon>0$ such that $L_{T} y-L_{T-1} y \geq \epsilon y$, or $L_{T} y \geq \epsilon y+L_{T-1} y=\left(\epsilon+r\left(L_{T-1}\right)\right) y$. By Theorem 2.3, $r\left(L_{T}\right)>r\left(L_{T-1}\right)$.

We obtain a corresponding result for the smallest positive eigenvalue, $\lambda_{0}$, of the eigenvalue problem (1.1), (1.2)

COROLLARY 3.2 Assume $p_{i_{0}}\left(T_{0}\right)>0$ for some $i_{0}, T_{0}$ such that $T_{0} \geq k-i_{0}$. Then the smallest positive eigenvalue, $\lambda_{0}(T)$, for the eigenvalue problem (1.1), (1.2) decreases for $T>T_{0}$. If, in addition, there exist $i_{1}, T_{1}$ such that $T_{1} \geq \min \left\{k-i_{1}, T_{0}+1\right\}$ and $p_{i_{1}}\left(T_{1}\right)>0$, then $\lambda_{0}\left(T_{1}\right)<\lambda_{0}\left(T_{0}\right)$.

PROOF. By Theorem 3.1, $r\left(L_{T_{0}}\right)>0$ and $r\left(L_{T_{0}}\right)$ is an eigenvalue of $L_{T_{0}}$. From the correspondence between eigenpairs of the eigenvalue problem and $L_{T_{0}}$, we see that $1 / r\left(L_{T_{0}}\right)$ is the smallest positive eigenvalue of (1.1), (1.2). Since $r\left(L_{T}\right)$ is increasing, it follows that $\lambda_{0}(T)=1 / r\left(L_{T}\right)$ is decreasing for $T>T_{0}$. Finally, $r\left(L_{T_{1}-1}\right)<r\left(L_{T_{1}}\right)$ by the previous theorem, and hence $\lambda_{0}\left(T_{0}\right) \geq$ $\lambda_{0}\left(T_{1}-1\right)>\lambda_{0}\left(T_{1}\right)$.

We now shall concentrate on the characterization of the first focal point of the boundary value problem and corresponding results.

THEOREM 3.3 Assume $p_{t_{0}}(T)>0$ for some $T \geq k-i_{0}$. Suppose that the boundary value problem (1.3), (1.2) has a nontrivial solution $y \in K_{T}$ (again, we mean $\left.y\right|_{[0, T+k-1]} \in K_{T}$ ). Then $T$ is the first focal point.

PROOF. Our first step will be to show $r\left(L_{T}\right)=1$. Now $L_{T} y=y$ implies $r\left(L_{T}\right) \geq 1$. Let $u \in K_{T}$ be an eigenvector corresponding to $r\left(L_{T}\right)$. We have shown in the proof of Theorem 3.1 that $y \in K_{T}^{\circ}$. Choose $\alpha$ maximal so that $y \geq \alpha u$. Then $y=L_{T} y \geq \alpha L_{T} u=\alpha r\left(L_{T}\right) u$. By the maximality of $\alpha, r\left(L_{T}\right) \leq 1$. Hence, $r\left(L_{T}\right)=1$. 
Finally, if the first focal point $\eta<T$, then $r\left(L_{\eta}\right) \geq 1$. But Theorem 3.1 shows that $r\left(L_{\eta}\right)<$ $r\left(L_{T}\right)$, contradicting $\mathrm{r}\left(L_{T}\right)=1$.

We are also interested in the uniqueness of the function corresponding to the first focal point. If conditions can be placed on the $p_{\imath}$ 's so that $L_{T}$ is $u_{0}$-positive with respect to $K_{T}$, then Krasnosel'skir's Theorem 2.4 can be applied. In this direction, then, suppose that $p_{k-1}(t)>0$ for $t \in[0, T]$. Since $K_{T}^{\circ}$ is nonempty, $K_{T}$ is reproducing. To show $L_{T}$ is $u_{0}$-positive, it is sufficient to show $L_{T}\left(K_{T} \backslash\{0\}\right) \subseteq K_{T}^{\circ}$.

Let $z \in K_{T} \backslash\{0\}$. First $\Delta^{k-1} z(t)>0$ for some $t \in[0, T]$; otherwise, $z$ would be the trivial solution. Then for some $s_{0} \in[0, T]$,

$$
\Delta L_{T} z(t)=\sum_{s=0}^{T} \Delta G_{T}(t, s) \sum_{i=0}^{k-1} p_{i}(s) \Delta^{2} z(s) \geq \Delta G_{T}\left(t, s_{0}\right) \sum_{i=0}^{k-1} p_{i}\left(s_{0}\right) \Delta^{i} z\left(s_{0}\right)>0
$$

for $t \in[k-j, T+k-1-j], 0 \leq j \leq k-1$. Therefore, $L_{T} z \in K_{T}^{\circ}$ for all $z \in K_{T} \backslash\{0\}$, and $L_{T}$ is $u_{0}$-positive.

Now suppose, in addition, that (1.3), (1.2) has a nontrivial solution $y \in K_{T}$. Note that by Theorem 3.3, $T$ is the first focal point. An application of Theorem 2.4 shows that $y$ is unique up to scalar multiple.

Finally, the requirement that $p_{k-1}$ be strictly positive can be replaced by other similar conditions. For example, if $p_{k-1}$ is identically zero, we could require $p_{k-2}>0$ and change our interval to $[0, T+k-2]$ in the definition of $B_{T}$.

However, under weaker conditions we can be certain that nontrivial solutions in $K_{T}$ are actually in $K_{T}^{\circ}$. This condition is the key to show uniqueness.

THEOREM 3.4 Let $p_{i_{0}}(T)>0$ for some $T \geq k-i_{0}$ and let $y \in K_{T}$ be a nontrivial solution of (1.3), (1.2). Then $y$ is unique up to scalar multiple.

PROOF. Since $y \in K_{T}$ is a solution to the boundary value problem (1.3), (1.2), then $y=L_{T} y$. Let $z$ also be a solution of the boundary value problem (1.3), (1.2) and assume $-z \notin K_{T}$. Choose $\alpha$ maximal so that $y \geq \alpha z$. We know that $\alpha>0$ since $y \in K_{T}^{\circ}$ by earlier arguments. Suppose $y-\alpha z \neq 0$. From previous work we know that $L_{T} y-\alpha L_{T} z \in K_{T}^{\circ}$. Then there is an $\epsilon>0$ so that $L_{T} y-\alpha L_{T} z \geq \epsilon z$. Hence $y-\alpha z \geq \epsilon z$ implies $y \geq(\alpha+\epsilon) z$, which contradicts the maximality of $\alpha$.

The next two theorems will examine conditions which guarantee that the first focal point of equation (1.3) is greater than or equal to $T$.

THEOREM 3.5 Assume that $p_{i_{0}}(T)>0$ for some $T \geq k-i_{0}$. If the smallest positive eigenvalue $\lambda_{0}(T)$ of (1.1), (1.2) satisfies $\lambda_{0}(T) \geq 1$, then the first focal point $\eta \geq T$.

PROOF. Suppose, on the contrary, that $\eta<T$. By Corollary 3.2, $\lambda_{0}(\eta)>\lambda_{0}(T)$. Then $\lambda_{0}(\eta)>1$ since $\lambda_{0}(T) \geq 1$. This says $r\left(L_{\eta}\right)=1 / \lambda_{0}(\eta)<1$. But for $z$, a solution of the boundary value problem, $L_{\eta} z=z$ implies $r\left(L_{\eta}\right) \geq 1$, which is a contradiction. Therefore, $\eta \geq T$.

THEOREM 3.6 Let $v$ be a nontrivial function in $K_{T}$ such that

$$
\sum_{s=0}^{T} G_{T}(t, s) \sum_{i=0}^{k-1} p_{i}(s) \Delta^{i} v(s) \leq v(t)
$$


with respect to $K_{T}$, where $p_{k-1}(t)>0$ for all $t \in[0, T]$. Then the first focal point $\eta \geq T$.

PROOF. Under the given hypothesis, we get that $L_{T} v \leq 1 v$ with respect to $K_{T}$. Let $\lambda_{0}(T)$ be the smallest positive eigenvalue of the eigenvalue problem (1.1), (1.2), and let $x$ be a corresponding eigenfunction in $K_{T}$. Then $r\left(L_{T}\right) x=L_{T} x=\left(1 / \lambda_{0}(T)\right) x$. By remarks preceding Theorem $3.4, L_{T}$ is $u_{0}$-positive, and it follows by Theorem 2.5 that $1 / \lambda_{0}(T) \leq 1$. Therefore, by Theorem 3.5 , the first focal point $\eta \geq T$.

As in the discussion preceding Theorem 3.4, the result still holds if we require that the last nontrivial coefficient function be strictly positive, and then modify the Banach space accordingly. These conditions guaranteed that $L_{T}$ was $u_{0}$-positive and allowed the application of Theorem 2.5 . However, we can relax the requirement of $u_{0}$-positivity, provided we add the condition $L_{T} v \neq 0$. To see this, consider the situation in Theorem 2.5 where $L \leq M$ and there are nonzero vectors $u_{1}, u_{2} \in K$ and scalars $\lambda_{1}, \lambda_{2}>0$ such that $L u_{1} \geq \lambda_{1} u_{1}$ and $M u_{2} \leq \lambda_{2} u_{2}$. In the proof of Theorem 2.5, $u_{0}$-positivity of $L$ was used to show $\epsilon_{0}=\sup \left\{\epsilon \mid L\left(u_{2}-\epsilon u_{1}\right) \geq 0\right\}>0$. In this case,

$$
0 \leq L\left(u_{2}-\epsilon_{0} u_{1}\right)=L u_{2}-\epsilon_{0} L u_{1} \leq M u_{2}-\epsilon_{0} \lambda_{1} u_{1} \leq \lambda_{2} u_{2}-\epsilon_{0} \lambda_{1} u_{1}=\lambda_{2}\left(u_{2}-\epsilon_{0} \frac{\lambda_{1}}{\lambda_{2}} u_{1}\right) .
$$

Hence $L\left(u_{2}-\epsilon_{0} \frac{\lambda_{1}}{\lambda_{2}} u_{1}\right) \in K_{T}$ implies $\lambda_{1} \leq \lambda_{2}$ by maximality of $\epsilon_{0}$.

Now suppose that $p_{i_{0}}(T)>0$ for some $T \geq k-i_{0}$, and that there is $v \in K_{T}$ such that $L_{T} v$ is nontrivial and $L_{T} v \leq v$. Using techniques similar to those in the proof of Theorem 3.1, it can be shown that $L_{T} v \in K_{T}^{\circ}$. If $x \in K_{T}$ is an eigenfunction for (1.1), (1.2) corresponding to $\lambda_{0}(T)$, then $\epsilon_{0}=\sup \left\{\epsilon \mid L_{T}(v-\epsilon x) \geq 0\right\}>0$. Hence, $1 \leq \lambda_{0}(T)$, and the first focal point $\eta \geq T$ from the same arguments used at the end of the proof of Theorem 3.6.

\section{REFERENCES}

1. HaRTMan, P. Difference equations: disconjugacy, principal solutions, Green's functions, complete monotonicity, Trans. Amer. Math. Soc. 246 (1978), 1-30.

2. Kelley, W. And Peterson, A. Difference Equations. Academic Press, New York, 1991.

3. Agarwal, R. P. ANd Lalli, B. S. Discrete: Polynomial interpolation, Green's functions, maximum principles, error bounds, and boundary value problems. Research Report 466, National University of Singapore, 1991.

4. Eloe, P. W. ANd Henderson, J. Comparison of eigenvalues for a class of two-point boundary value problems, Applicable Anal. 34 (1989), 25-34.

5. Eloe, P. W. ANd Henderson, J. Comparison of eigenvalues for a class of multipoint boundary value problems, Recent Trends in Ordinary Differential Equations. (to appear).

6. Gentry, R. D. AND Travis, C. C. Comparison of eigenvalues associated with linear differential equations of arbitrary order, Trans. Amer. Math. Soc. 223 (1976), 167-179.

7. Hankerson, D. AND Henderson, J. Positive solutions and extremal points for differential equations, Applicable Anal. 39 (1990), 193-207.

8. Hankerson, D. AND Peterson, A. Comparison theorems for eigenvalue problems for $n$th order differential equations, Proc. Amer. Math. Soc. 104 (1988), 1204-1211.

9. Hankerson, D. AND Peterson, A. Comparison of eigenvalues for focal point problems for $n$th order difference equations, Differential and Integral Equations 3 (1990), 363-380. 
10. KeEner, M. S. and Travis, C. C. Sturmian theory for a class of nonselfadjoint differential systems, Ann. Mat. Pura Appl. 123 (1980), 247-266.

11. Томаsтік, E. Comparison theorems for second order nonselfadjoint differential systems, SIAM J. Math. Anal. 14 (1983), 60-65.

12. Tомаsтік, E. Comparison theorems for conjugate points of $n$th order nonselfadjoint differential equations, Proc. Amer. Math. Soc. 96 (1986), 437-442.

13. Travis, C. C. Comparison of eigenvalues for linear differential equations of order $2 n$, Trans. Amer. Math. Soc. 177 (1973), 363-374.

14. Eloe, P. W. Criteria for right disfocality of linear difference equations, J. Math. Anal. Appl. 120 (1986), 610-621.

15. Eloe, P. W. and Henderson, J. Analogues of Fekete and Descartes systems of solutions for difference equations, Journal of Approximation Theory 59 (1989), 38-52.

16. HENDERSON, J. Focal boundary value problems for nonlinear difference equations, I, J. Math. Anal: Appl. 141 (1989), 559-567.

17. Henderson, J. Focal boundary value problems for nonlinear difference equations, II, J. Math. Anal. Appl. 141 (1989), 568-579.

18. Henderson, J. AND LEe, L. Continuous dependence and differentiation of solutions of finite difference equations, Internat. J. Math. Math. Sci. 14 (1991), 747-756.

19. Keener, M. S. And Travis, C. C. Positive cones and focal points for a class of $n$th order differential equations, Trans. Amer. Math. Soc. 237 (1978), 331-351.

20. Schmitt, K. AND Smith, H. L. Positive solutions and conjugate points for systems of differential equations, Nonlinear Anal. 2 (1978), 93-105.

21. Tomastik, E. Comparison theorems for focal points of systems of $n$th order nonselfadjoint differential equations, Rocky Mountain J. Math. 18 (1988), 1-11.

22. Krasnosel'skil̆, M. A. Positive Solutions of Operator Equations. Fizmatgiz, Moscow, 1962; English Translation P. Noordhoff Ltd. Groningen, The Netherlands, 1964.

23. KReǏn, M. G. AND RUtman, M. A. Linear operators leaving a cone invariant in a Banach space. In American Mathematical Society Translations, Series 1 Volume 10, pages 199-325. American Mathematical Society, Providence, R. I., 1962.

24. Deimling, K. Nonlinear Functional Analysis. Springer-Verlag, New York, 1985.

25. Peterson, A. Green's functions for $(k, n-k)$-boundary value problems for linear difference equations, J. Math. Anal. Appl. 124 (1987), 127-138. 


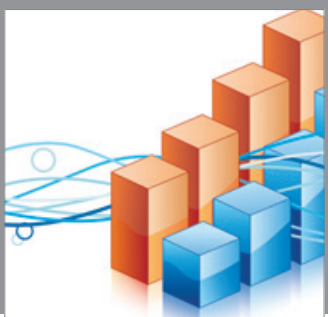

Advances in

Operations Research

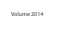

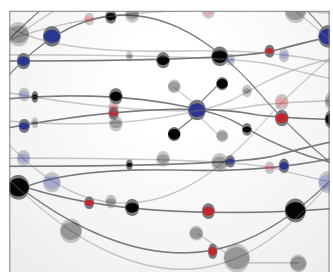

\section{The Scientific} World Journal
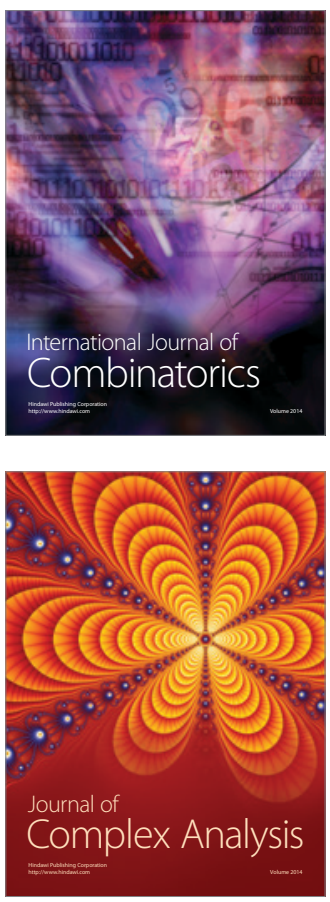

International Journal of

Mathematics and

Mathematical

Sciences
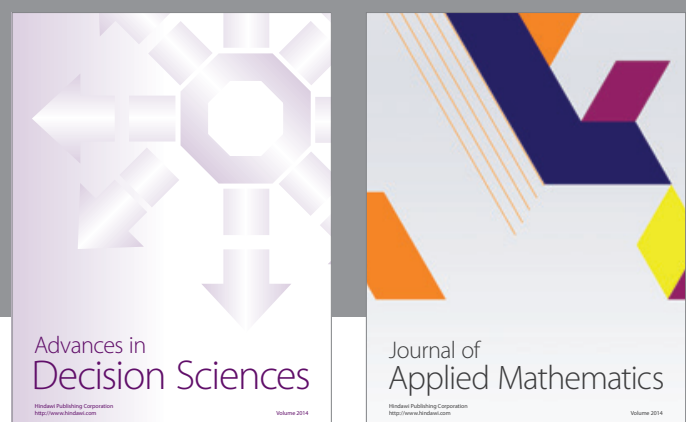

Journal of

Applied Mathematics
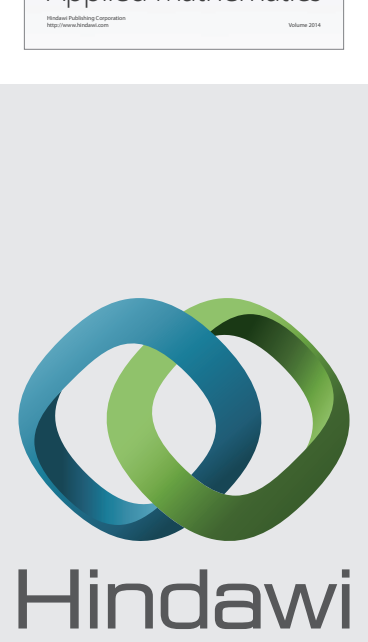

Submit your manuscripts at http://www.hindawi.com
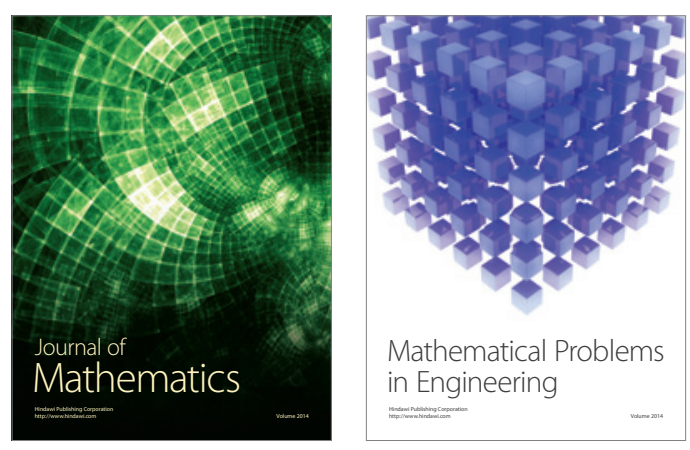

Mathematical Problems in Engineering
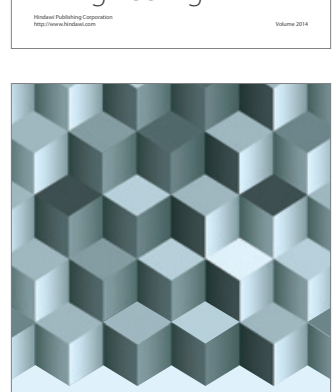

Journal of

Function Spaces
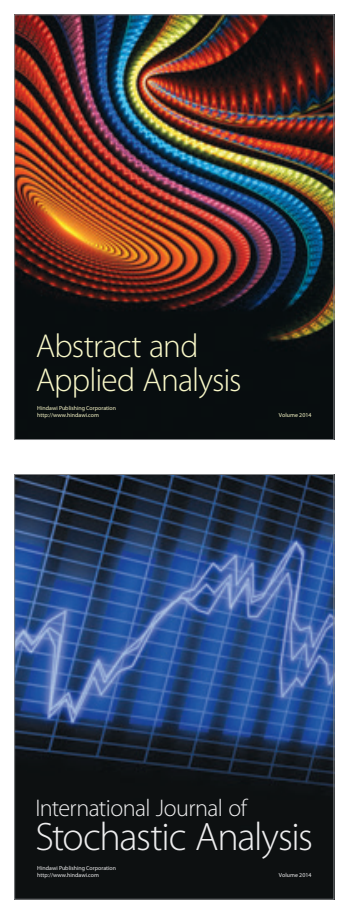

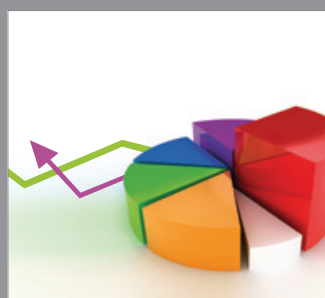

ournal of

Probability and Statistics

Promensencen
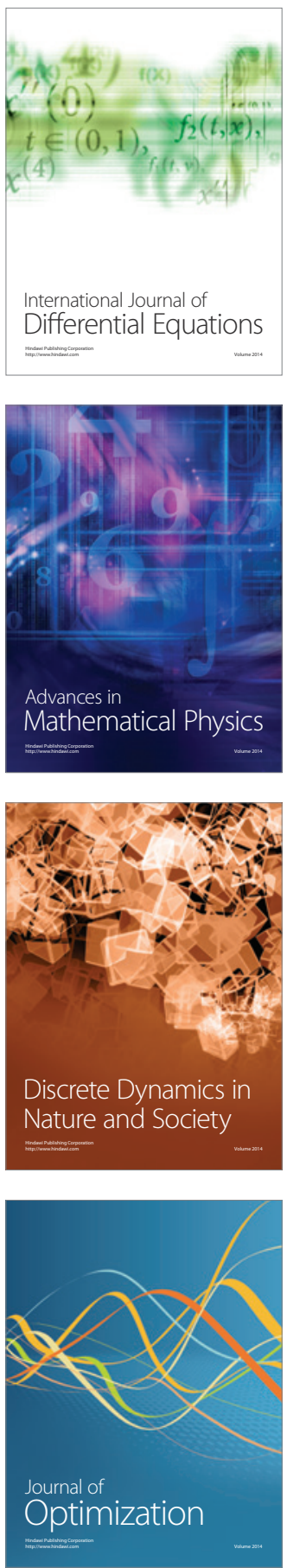